\title{
Wave farm modelling: harnessing Ireland's greatest energy resource
}

\section{Brendan Cahill}

Hydraulics and Maritime Research Centre, UCC

\section{Introduction}

Wave Energy Converters are being developed worldwide, including by a number of Irish companies, as a sustainable and environmentally-friendly means of generating electricity using the power of the ocean. By 2020 it is envisaged that hundreds of these devices will be deployed off the West Coast of Ireland in arrays known as Wave Farms and connected to the national electricity grid. The focus of my Ph.D. project, being carried out at the Hydraulics and Maritime Research Centre (HMRC) in UCC, is to create realistic models of these Wave Farms so that we can better understand their behaviour and ultimately optimise their performance.

\section{Why Wave Energy?}

Nearly $95 \%$ of the electricity consumed in Ireland is generated from fossil fuels such as gas, coal and oil. As reserves of these resources begin to dry up, leading to scarcity of supply and increases in cost, and the environmental concerns about carbon dioxide emissions become more pronounced, Ireland will need to develop more sustainable sources of energy to light our homes and keep our myriad of electrical appliances running. While we are already utilising renewable forms of electricity generation such as wind turbines and hydroelectric plants, our greatest natural energy resource resides in the waves that lash our coast, especially on the western seaboard.

Ireland is ideally located to capture Wave Energy. The prevailing South-West wind blowing across the Atlantic Ocean, generating waves, means that we are situated at the end of a vast, natural transmission line for wave power. A 2005 report by ESB International estimated that the theoretical Wave Energy resource off the Irish coast amounts to 525 terawatt hours (TWh) annually. In comparison, our total electricity requirement for the year 2008 came to $27.8 \mathrm{TWh}$. Harnessing even a tiny fraction of our natural power would go a long way to meeting our electricity needs. 


\section{WEC Arrays}

The devices that will be used to capture this energy are commonly known as Wave Energy Converters (WECs). To date there is no universally accepted design for a WEC. A quick internet search will provide examples of dozens of different devices with radically different shapes, sizes and operating principles. All these competing designs must be deployed in arrays to form Wave Farms. The reason for this is that when compared to a traditional power plant a typical WEC, operating in isolation is small, with the capacity to provide power for approximately 1,500 homes. As a result multiple devices will have to be placed at sea in close proximity to each other to produce a grid scale generation plant. This will present many challenges as it is likely that within an array the WECs will interact with each other by radiating waves outwards or by absorbing the energy destined for a neighbouring device. The aim of my research is to develop an understanding of this behaviour so that wave farms can be designed to extract the maximum amount of energy.

\section{Wave Field Modelling}

In order to investigate the performance of WEC arrays it is necessary to be able to describe the ocean waves that drive the operation of these devices. An integral part of my research involves the simulation of wave fields through computer modelling. Initially the ocean surface seems to be a chaotic place that would be difficult to model, with waves of varying size appearing to come from a range of angles at random intervals. However it is possible to recreate realistic, irregular wave profiles by simply adding together the correct combination of simple regular waves of different height, length and direction. This combination can be derived from previously developed mathematical formulae, or from the data provided by wave measurement buoys located offshore. This allows wave profiles to be generated for the all potential device locations within a Wave Farm, even when these points are separated by several kilometres. An example of this can be seen in Fig. 1 which illustrates the evolution of the water surface profile in a $50 \mathrm{~m} \times 50 \mathrm{~m}$ wave field at intervals of 10 seconds.

\section{Power Smoothing from WEC Arrays}

Investigating the power smoothing effect when WECs are placed in arrays is one application of ocean surface modelling at Wave Farm scale. Because of the irregular nature of real sea waves the incoming energy at any point is not steady. Significant fluctuations can be seen - the power generated by a device can go from practically zero to a dramatic spike several times larger than the average value in a matter of seconds, as seen with the red line in Fig. 2 (a) on p.24. These fluctuations are unacceptable if the power captured from waves is to be fed into the electricity grid and methods of smoothing out the supply from WECs must be developed. To date a number of solutions to this problem have 


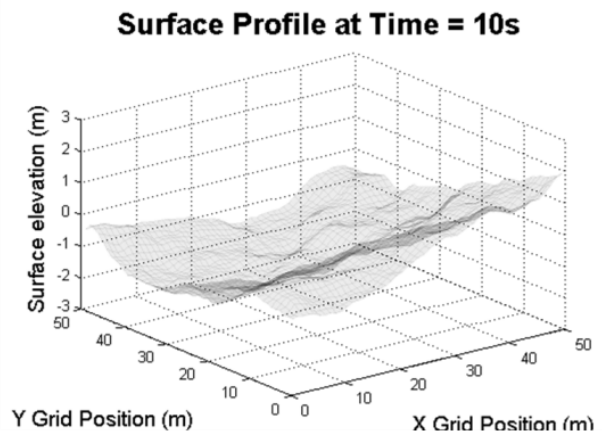

Surface Profile at Time $=30 \mathrm{~s}$

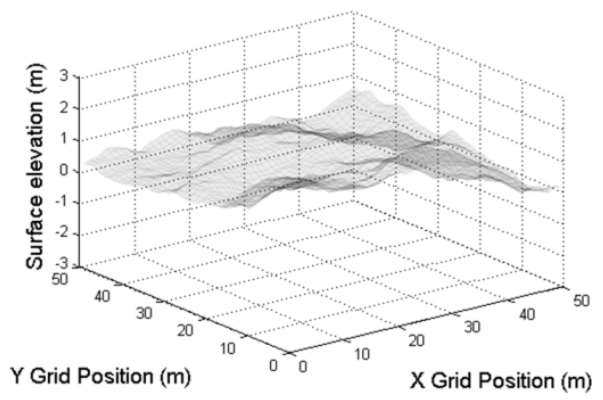

Surface Profile at Time $=20 \mathrm{~s}$

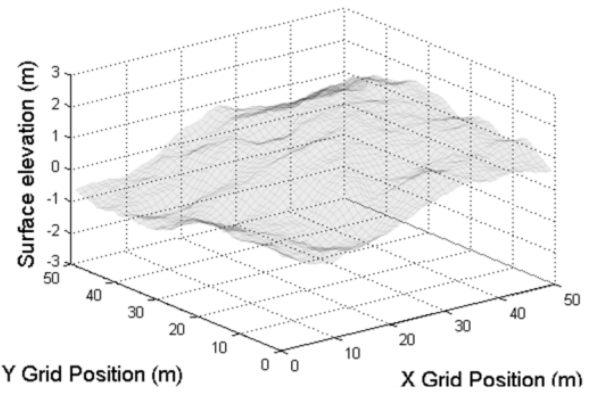

Surface Profile at Time $=40 \mathrm{~s}$

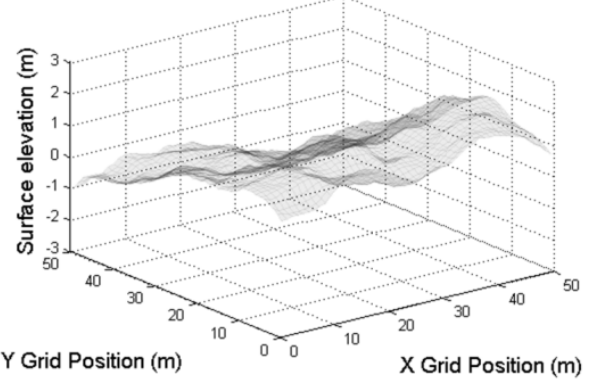

Figure 1: Simulated Wave Field at 10 second intervals

been suggested. It may be possible to temporarily store the energy generated by WECs using batteries or flywheels, allowing the output power to be regulated, while the motion of devices can be controlled so that the resulting output power is less intermittent. The approach I've taken is to examine if the inherent variability of the wave fields where farms will be situated can be utilised so that the total power output from the array is smoother, i.e., will the power peaks of a portion of the devices be cancelled out by the troughs of others?

\section{Results}

Surprisingly, neither changing the distance between the individual devices nor changing the overall layout of the array had much effect on the smoothness of the output power, which can be quantified by calculating the standard deviation of the total power produced by the farm over the time period being investigated. Of greater influence is the number of WECs in a farm. In Fig. 2 (b) it can be seen that the total output from an array of 25 devices showed approximately one-fifth of the variability of a device operating in isolation in similar wave conditions. It is also worth noting that the very severe peaks and troughs in output for a single WEC have been eliminated, see Fig. 1 (a). This result is a promising one in terms of the future deployment of large scale, commercial farms. It is envisaged that the first WEC arrays will be of a similar size, or possibly even larger, than the farms assessed in this work. The outputs from this study indicate that a level of smoothing will 
(a)

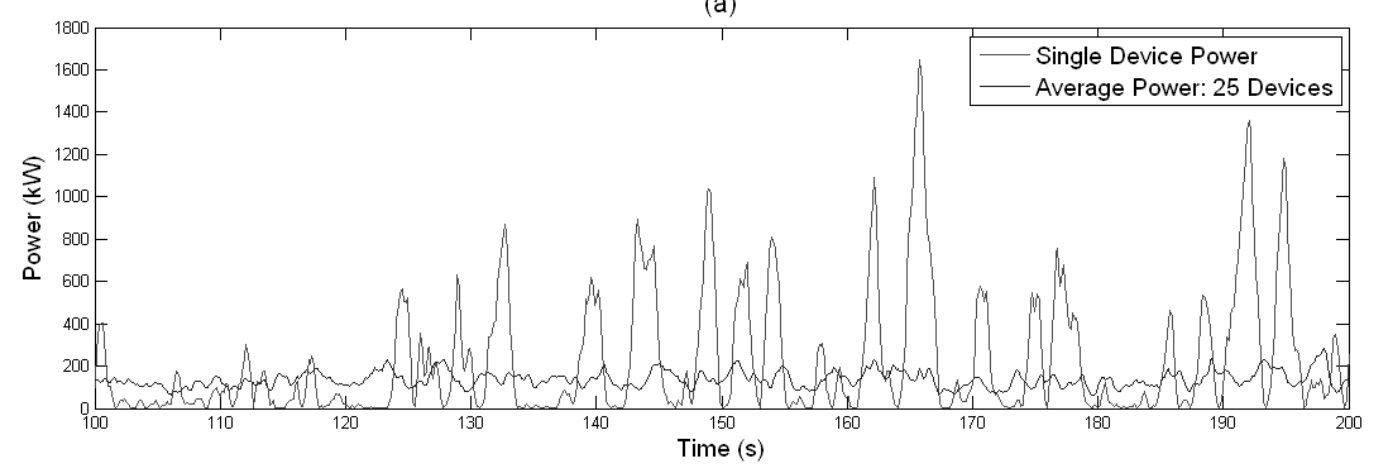

(b)

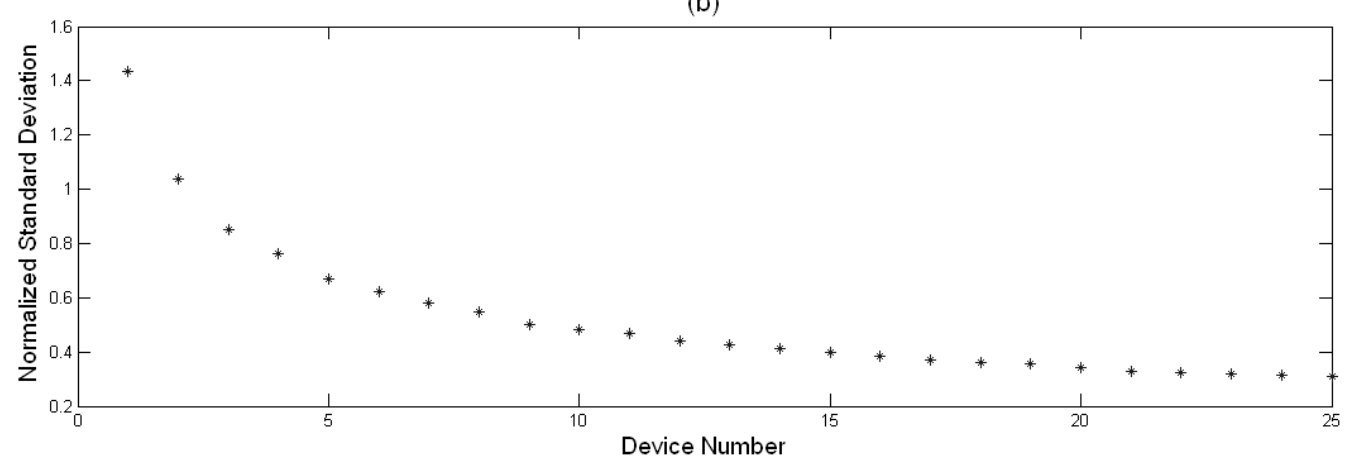

Figure 2: (a) Power output from a single device (red) and average device output from a farm of 25 WECs (blue); (b) Decreasing variability with increasing number of devices in a Wave Farm.

be inbuilt in these developments, as the total aggregate power will be far less intermittent that the yield from a single device. This inherent smoothing effect will ensure that the power produced by Wave Farms will be much more grid-friendly and should reduce the need for incorporating methods of short-term energy storage into WEC array designs.

\section{Future Work}

I hope to build on this work by creating more realistic models of WEC operation and include interactions between the devices in an array. It would also be interesting to use this approach to study geographical smoothing; i.e., distributing the locations of potential Wave Farm sites along the coast so that fluctuations in local meteorological conditions have less influence on overall power production. Finally, I will investigate the accuracy of these numerical models by carrying out testing of small scale models in the $25 \mathrm{~m} \mathrm{x} 18 \mathrm{~m}$ wave tank at the HMRC.

The author wishes to thank Dr. Tony Lewis for his supervision and assistance and the Irish Research Council for Science, Engineering and Technology (IRCSET) for funding this research. 